\title{
Selección de soportes magnéticos para la inmovilización de Ureasa
}

\section{Magnetic supports selection for Urease inmobilization}

\author{
Hugo A Rojas*§, José J Martínez*, Annie Y. Vargas* \\ * Escuela de Ciencias Químicas. Facultad de Ciencias. Grupo de Catálisis (GC-UPTC). Universidad \\ Pedagógica y Tecnológica de Colombia, Avenida Central del Norte, Vía Paipa, Tunja, Boyacá- \\ Colombia.\$hugo.rojas@uptc.edu.co,jose.martinez@uptc.edu.co,annie.vargas@uptc.edu.co
}

Recibido: 16 de Octubre de 2013 - Aceptado: Octubre 20 de 2014

\section{Resumen}

Sólidos magnéticos tipo $\mathrm{Fe}_{3} \mathrm{O}_{4}, \mathrm{Fe}_{3} \mathrm{O}_{4}-\mathrm{NH}_{2}, \mathrm{Fe}_{3} \mathrm{O}_{4} @ \mathrm{SiO}_{2}$ y $\mathrm{Fe}_{3} \mathrm{O}_{4} @ \mathrm{SiO}_{2}-\mathrm{NH}$ se usaron como soportes en la inmovilización de la ureasa, estos sólidos fue caracterizados por análisis de fisisorción de $\mathrm{N} 2$ a $77 \mathrm{~K}$, curvas de magnetización y FTIR. La actividad enzimática se evaluó por descomposición de la urea. Los sólidos presentan características magnéticas que les permite fácilmente ser separados. La enzima inmovilizada mejoró notablemente su actividad enzimática comparada con la enzima libre. Para los sólidos $\mathrm{Fe}_{3} \mathrm{O}_{4}-\mathrm{NH}_{2}, \mathrm{y} \mathrm{Fe} \mathrm{O}_{3} @ \mathrm{SiO}_{2}-\mathrm{NH}_{2}$ se observó una mayor cantidad de enzima acoplada.

Palabras clave: APTES, inmovilización, magnetita, soportes, ureasa.

\begin{abstract}
$\mathrm{Fe}_{3} \mathrm{O}_{4}, \mathrm{Fe}_{3} \mathrm{O}_{4}-\mathrm{NH}_{2}, \mathrm{Fe}_{3} \mathrm{O}_{4} @ \mathrm{SiO}_{2}$ y Fe $\mathrm{O}_{4} @ \mathrm{SiO}_{2}-\mathrm{NH}_{2}$ as magnetic supports were studied for the urease immobilization. The solids were characterized by $\mathrm{N}_{2}$-physical adsorption at $77 \mathrm{~K}$, magnetization curves and FTIR. Activity assays were evaluated by urea decomposition. The magnetic properties of solids allow an easy separation. The immobilized enzyme improved the enzymatic activity compared to the free enzyme. In $\mathrm{Fe}_{3} \mathrm{O}_{4}-\mathrm{NH}_{2}$ and $\mathrm{Fe}_{3} \mathrm{O}_{4} @ \mathrm{SiO}_{2}-\mathrm{NH}_{2}$ was observed allows a greater amount of enzyme coupled.
\end{abstract}

Keywords: APTES, immobilization, magnetite, supports, urease 


\section{Introducción}

Las enzimas poseen excelente actividad catalítica, expresan mayor regio y enantioselectividad (Demir, A. S. et al., 2011), mayor especificidad (Tomotani and Vitolo, 2006), permiten realizar procesos a bajas temperaturas, generan menor cantidad de desechos tóxicos, contaminantes, emisiones y subproductos en comparación con los procesos químicos convencionales, por lo que son ampliamente utilizadas a nivel industrial (Tomotani, E. J. et al., 2007). Así las enzimas son parte fundamental en la biocatálisis, tecnología clave para la síntesis orgánica moderna, en especial de productos químicos finos y productos farmacéuticos.(Bolivar et al., 2013)

A pesar de esto, las enzimas sufren de numerosos problemas al momento de la aplicación práctica, como pérdida de la estabilidad frente a $\mathrm{pH}$, calor $\mathrm{u}$ otros factores, difícil recuperación y re-uso en un sistema de reacción, que ocasionan altos costos de operación. Estas deficiencias evitan la explotación eficaz de las ventajas técnicas y económicas de las enzimas, especialmente en una amplia variedad de reacciones de bio-transformación (Demir et al., 2011, Cao et al., 2012)). Hay técnicas que permiten mejorar la estabilidad de la enzima tales como la inmovilización, la modificación enzimática, ingeniería de proteínas, entre las cuales la inmovilización es el método más frecuentemente utilizado para mejorar las características de estas (Cao, et al., 2012).

En los últimos años, se han utilizado nanopartículas como soportes para la inmovilización de enzimas, puesto que proporcionan una gran área superficial, mejorando la eficiencia catalítica de las enzimas inmovilizadas, sin embargo la recuperación de la enzima inmovilizada en nano partículas es a menudo limitada (Hong et al., 2009, Ansari and Husain, 2012, Cao et al., 2012, Ashtari et al., 2012, Jiang et al., 2009). Recientemente, el uso de nanopartículas magnéticas ha tomado gran auge debido a sus interesantes propiedades químicas y físicas tales como la estabilidad, biocompatibilidad y superparamagnetismo, baja toxicidad, reducción de la auto agregación y fácil separabilidad de la mezcla de reacción mediante la aplicación de un campo magnético (Netto, C. G. C. M. et al., 2013). Poseen además una amplia gama de aplicaciones en la inmovilización de células y enzimas, sistemas de bioseparación, inmunoensayos, administración de fármacos $\mathrm{y}$ biosensores (Ashtari, K. et al., 2012).

Las nanopartículas magnéticas pueden ser funcionalizados por grupos especiales (por ejemplo $-\mathrm{OH},-\mathrm{COOH},-\mathrm{NH}_{2}$ ) (Feng, B. et al., 2008) apto para otras modificaciones por la unión de varias moléculas bioactivas en distintas aplicaciones (Cao, M. et al., 2012, Zhao, G. et al., 2011).

Sin embargo, una de las desventajas principales de las nanopartículas magnéticas radica en que no son estables por lo cual son fácilmente oxidables $\mathrm{y}$ las hace vulnerables a perder el magnetismo. Otras de las desventajas es que son propensas a agregarse, muestran pobre dispersión en agua y en disolventes orgánicos. Así las estrategias de protección, y conservación de estas nanopartículas magnéticas durante y después de la síntesis, es la estabilización química a partir de recubrimientos en capas con compuestos orgánicos o inorgánicos. Para el caso de recubrimientos inorgánicos se utilizan por lo general sistemas porosos para estabilizar las partículas magnéticas, como la sílice. Las nanopartículas magnéticas recubiertas de sílice proporcionan muchos grupos reactivos en la superficie, a través de grupos silanoles los cuales son empleados directamente en la posterior funcionalización de la superficie.

Una enzima modelo para la inmovilización de enzimas es la ureasa, altamente eficaz para la hidrólisis de la úrea. La principal aplicación de la ureasa se encuentra en el análisis del contenido de úrea en sangre, tratamiento en bebidas alcohólicas y desarrollo de sensores (Luo and Fu, 2010, Sahoo et al., 2011, Krishna et al., 2011), entre otras. Sin embargo, la búsqueda de un medio sencillo para separar la enzima del proceso, permitiría el reuso de la enzima. En este sentido, el uso de nanopartículas magnéticas sería muy eficaz para inmovilizar la enzima y facilitar su separación por uso de un campo magnético externo (Tartaj et al., 2005). En cada una de las aplicaciones anteriores, 
la inmovilización de la enzima se constituye en un importante reto científico.

Algunos resultados muestran que es posible inmovilizar ureasa en nano-partículas magnéticas, obteniendo un aumento en el tiempo de almacenamiento por períodos más largos y en su estabilidad térmica, así mismo la actividad de la enzima inmovilizada no es afectada por el cambio en rangos amplios de $\mathrm{pH}$, sin perder su actividad catalítica inicial (Sahoo et al., 2011). En este trabajo se pretendió evaluar la inmovilización de ureasa en partículas de $\mathrm{Fe}_{3} \mathrm{O}_{4}$ modificadas con APTES o sílice con el fin de seleccionar dentro de estos soportes magnéticos cual permitiría una mayor inmovilización de la enzima sin alterar su comportamiento catalítico.

\section{Experimental}

\subsection{Materiales}

La enzima utilizada es la ureasa de Canavalia ensiformis (Jack bean, EC 3.5.1.5, 5 UI). Los cloruros de hierro $\mathrm{FeCl}_{3} \cdot 6 \mathrm{H}_{2} \mathrm{O}, \mathrm{FeCl} 2.4 \mathrm{H}_{2} \mathrm{O}$, aminopropiltrietoxisilano al 99\% (APTES), tetraetilortosilicato (TEOS), purpura de bromocresol, son de Sigma aldrich, NaH2PO4 y $\mathrm{Na}_{2} \mathrm{HPO}_{4}$ de Mobs Labs, $\mathrm{NaOH}$ de Panreac , $\mathrm{HCl}$ de Riedel-de Haen, $\mathrm{NH}_{4} \mathrm{OH}$ de E.M, etanol de J.T Baker y agua desionizada.

\subsection{Síntesis de las nanopartículas de $\mathrm{Fe}_{3} \mathrm{O}_{4}$}

Las nanoparticulas magnéticas fueron sintetizadas mediante una co-precipitación alcalina de sales de hierro (Soo Y., 1996). El procedimiento de preparación inició con la disolución de $\mathrm{FeCl}_{3} \cdot 6 \mathrm{H}_{2} \mathrm{O}$ y $\mathrm{FeCl}_{2} \cdot 4 \mathrm{H}_{2} \mathrm{O}$ (Acros $99 \%$ ) en una mezcla de agua (burbujeada previamente con $\mathrm{N}_{2}$ ) y ácido clorhídrico, bajo agitación mecánica hasta total disolución, luego se adicionó hidróxido de sodio con agitación continua, y se dejó decantar, se le acercó un imán externo y se comprobó su carácter paramagnético, al precipitado obtenido se le adicionó $\mathrm{HCl}$ para neutralizar los cargas aniónicas de las nanopartículas. Se lavó repetidas veces con agua desionizada y se secó al vacío a $333 \mathrm{~K}$.

\subsection{Síntesis de $\mathrm{Fe}_{3} \mathrm{O}_{4} @ \mathrm{SiO}_{2}$}

El procedimiento de encapsulación de las microesferas magnéticas con $\mathrm{SiO}_{2}$ se realizó a través del método de Stöber (Luo et al., 2010). En el que se tomó las nanopartículas de $\mathrm{Fe}_{3} \mathrm{O}_{4}$ sintetizadas previamente y se dispersaron en una mezcla de etanol, agua desionizada y solución de amoniaco acuoso concentrado $(28 \%$ en peso). Esta mezcla se llevó a ultrasonido por 1h. Posteriormente tetraetil ortosilicato (TEOS) como fuente de sílice fue adicionada gota a gota a la mezcla, dejando en agitación por $3 \mathrm{~h}$, bajo atmosfera de nitrógeno. Los productos fueron recogidos, lavados con agua desionizada y secados a vacío a $333 \mathrm{~K}$. La relación entre las partículas magnéticas y la fuente de sílice se mantuvo 1:1.

\subsection{Funcionalización con APTES de Fe3O4 y Fe3O4@SiO2}

Los sólidos $\mathrm{Fe}_{3} \mathrm{O}_{4} \quad \mathrm{y} \quad \mathrm{Fe}_{3} \mathrm{O}_{4} @ \mathrm{SiO}_{2} \quad$ se funcionalizaron con APTES siguiendo el método propuesto por Diez, et al., (Díez et al., 2012) en donde las nanopartículas magnetitas se dispersaron por sonicación en una mezcla de etanol y agua, posteriormente se le adiciona 3-aminopropiltrietoxisilano (APTES), y se agitó mecánicamente por $7 \mathrm{~h}$ en atmosfera de nitrógeno, luego se decantaron magnéticamente, se lavaron con una mezcla de agua-etanol, y secaron con una a temperatura ambiente, para la obtención de $\mathrm{Fe}_{3} \mathrm{O}_{4}$ NH2 y Fe3O4@ $\mathrm{SiO}_{2}-\mathrm{NH}_{2}$, respectivamente.

\subsection{Inmovilización de ureasa}

Para la inmovilización de la ureasa, la enzima se disolvió en una solución de buffer fosfatos manteniendo el pH en 5,8, a la cual se le adicionó una proporción de cada soporte magnético manejando una relación $1: 2$, se agitó por $24 \mathrm{~h}$ y se mantuvo el sistema a $4{ }^{\circ} \mathrm{C}$, para lograr esta temperatura el agitador orbital se mantuvo en un refrigerador convencional. En este proceso se espera que la enzima se impregne en el soporte. Posteriormente, se centrifugó y se lavó varias veces el soporte con solución buffer fosfatos, para retirar la enzima residual. 


\subsection{Evaluación de la proteína acoplada}

El primer sobrenadante obtenido, se utilizó para la determinación de proteína por el método de Bradford utilizando azul de Coomassie G250 a 595nm en un espectrofotómetro UV-VIS GBC-911.

\subsection{Evaluación de la actividad enzimática}

Se evaluó la actividad enzimática por el método colorimétrico basado en la hidrólisis de la urea, monitoreando el cambio de $\mathrm{pH}$ con tinte púrpura de bromocresol (Zong, J. et al., 2011). La reacción enzimática se monitorizó siguiendo la absorción de colorante a $588 \mathrm{~nm}$ en un espectrofotómetro UV-VIS GBC-911.

\subsection{Caracterización de los sólidos}

La evaluación magnética de los sólidos se realizó a temperatura ambiente $\left(19^{\circ} \mathrm{C}\right)$ en un magnetómetro Versalab. La superficie específica, el diámetro de poro y el volumen de poro de los sólidos preparados fueron determinados desde las isotermas de adsorción - desorción de N2 a 77K obtenidas en un Micromeritics ASAP 2020. Análisis por Infrarrojo (FTIR) fueron realizados en un Nicolet 6300.

\section{Resultados y discusión}

\subsection{Caracterización de los sólidos}

Las isotermas de adsorción-desorción se muestran en la figura 1 y los resultados de superficie específica, tamaño y volumen de poro se pueden observar en la tabla 1. Las isotermas obtenidas de adsorción son de tipo IV, características de sólidos mesoporosos, sin embargo los distintos ciclo de histéresis sugieren que la incorporación del APTES y de la sílice a las partículas de $\mathrm{Fe}_{3} \mathrm{O}_{4}$ modifican las propiedades texturales. Las partículas magnéticas de $\mathrm{Fe}_{3} \mathrm{O}_{4}$ presentan una baja área superficial y la superficie aumenta a medida que se funcionaliza con APTES o se incorpora sílice. El incremento del valor de área superficial a.

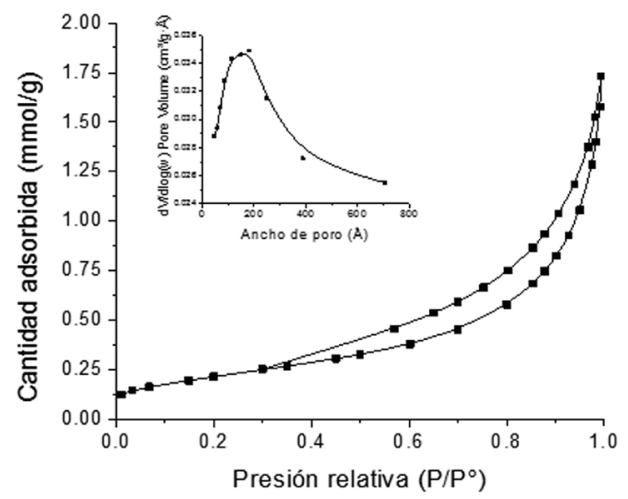

C.

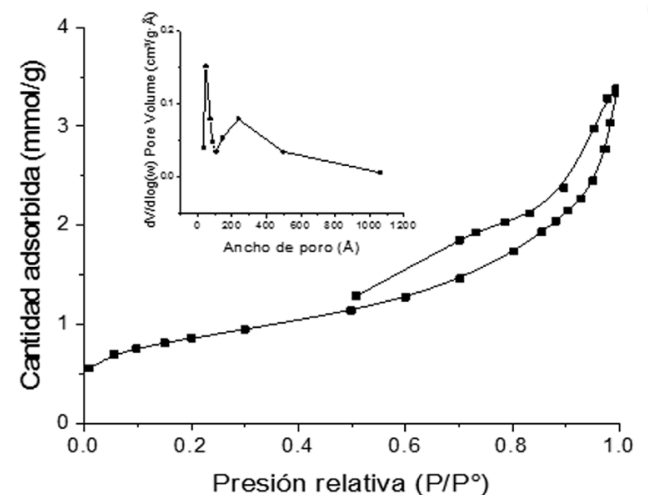

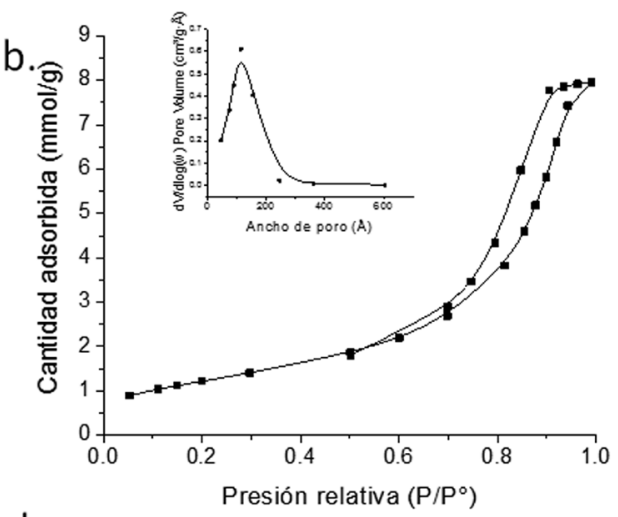

d.

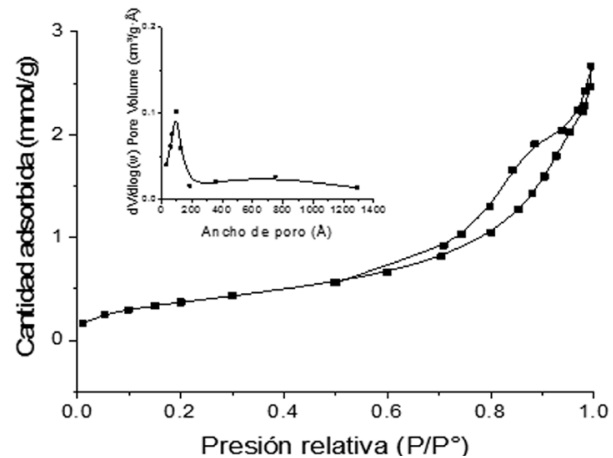

Figura 1. Isotermas de adsorción-desorción de nitrógeno y distribución de tamaño de poros de los soportes magnéticos (a) $\mathrm{Fe}_{3} \mathrm{O}_{4}$, (b) $\mathrm{Fe}_{3} \mathrm{O}_{4}-\mathrm{NH}_{2}$, (b) $\mathrm{Fe}_{3} \mathrm{O}_{4} @ \mathrm{SiO}_{2}$, (d) $\mathrm{Fe}_{3} \mathrm{O}_{4} @ \mathrm{SiO}_{2}-\mathrm{NHz}$. 
Tabla 1. Propiedades texturales de los sólidos estudiados.

\begin{tabular}{cccc}
\hline Sólidos & SBET, $\boldsymbol{m}^{2} / \boldsymbol{g}$ & Vp, $\boldsymbol{m L} / \boldsymbol{g}$ & dporo, nm \\
\hline $\mathrm{Fe}_{3} \mathrm{O}_{4}$ & 17 & 0,06 & 13,8 \\
$\mathrm{Fe}_{3} \mathrm{O}_{4} @ \mathrm{SiO}_{2}$ & 64 & 0,11 & 7,2 \\
$\mathrm{Fe}_{3} \mathrm{O}_{4}-\mathrm{NH}_{2}$ & 99 & 0,28 & 11,5 \\
$\mathrm{Fe}_{3} \mathrm{O}_{4} @ \mathrm{SiO}_{2}-\mathrm{NH}_{2}$ & 52 & 0,14 & 11,1 \\
\hline $\mathrm{S}_{B E T}=$ superficie especifica BET, $\mathrm{Vp}=$ volumen de poro, $d_{\text {poro }}=$ diámetro de poro.
\end{tabular}

(SBET) en $\mathrm{Fe}_{3} \mathrm{O}_{4} \mathrm{NH}_{2}$ puede explicarse debido a que el APTES sirve de agente dispersante de las partículas de $\mathrm{Fe}_{3} \mathrm{O}_{4}$, lo que ocasiona un cambio de la distribución de poros como se observa en la figura $1 \mathrm{~b}$. El aumento de la superficie específica y la distribución de poros bimodal en $\mathrm{SiO}_{2} @$ $\mathrm{Fe}_{3} \mathrm{O}_{4}$ (figura 1c) se explica por un recubrimiento parcial de las partículas de $\mathrm{Fe}_{3} \mathrm{O}_{4}$ con sílice. $\mathrm{Al}$ incorporar el APTES a la superficie de $\mathrm{SiO}_{2} @$ $\mathrm{Fe}_{3} \mathrm{O}_{4}\left(\mathrm{Fe}_{3} \mathrm{O}_{4} @ \mathrm{SiO}_{2}-\mathrm{NH}_{2}\right.$ : figura 1d), es posible que se dispersen las partículas de $\mathrm{Fe}_{3} \mathrm{O}_{4}$ o se ocluyan algunos poros de la sílice. En consecuencia, el modo de actuar del APTES dependerá de la superficie donde se ancla.

En la figura 2 se observan las curvas de magnetización en función del campo magnético de los sólidos estudiados. La dependencia del campo magnético aplicado para estos sistemas confirma la respuesta ferromagnética de estos materiales. Se observa que todas las curvas pasan por el origen, lo que se traduce en que se pueden volver a dispersar fácilmente cuando el campo magnético aplicado es retirado. Por tanto explica la razón por la cual en los sólidos de $\mathrm{Fe}_{3} \mathrm{O}_{4} @ \mathrm{SiO}_{2}$ y Fe $\mathrm{F}_{3} @ \mathrm{SiO}_{2}-\mathrm{NH}_{2}$ donde existe un posible recubrimiento de sílice de las partículas magnéticas, esta respuesta magnética permanece, pudiendo ser fácilmente separadas con un imán. La respuesta magnética de la magnetita funcionalizada con APTES $\left(\mathrm{Fe}_{3} \mathrm{O}_{4}-\mathrm{NH}_{2}\right)$ no cae tan bruscamente como las magnetitas recubiertas con sílice, dado que la funcionalización afecta solamente la superficie de estas partículas.

La figura 3 muestra los espectros para los soportes obtenidos. La banda de absorción en $590 \mathrm{~cm}^{-1}$ en la figura 3a ha sido asociada al enlace Fe-O de la magnetita (Waldron, 1955, Ma et al., 2003) Las señales observadas en $1625 \mathrm{~cm} 1$, se atribuyen a las vibraciones de los enlaces N-H (Ma et al., 2003) lo que permite afirmar que se obtuvo partículas magnéticas funcionalizadas con APTES (Fig. 3 (b) y 3 (c)). El encapsulamiento de la magnetita con $\mathrm{SiO}_{2}$ (Fig. 3c) se presenta con la absorción de los grupos silanoles en la superficie de las partículas magnéticas y se observa en las bandas entre 1111 y $1049 \mathrm{~cm}^{-1}$ que corresponden a los grupos $\mathrm{SiO}-\mathrm{H}$ y Si-O-Si. Las bandas de absorción entre 750 y 900

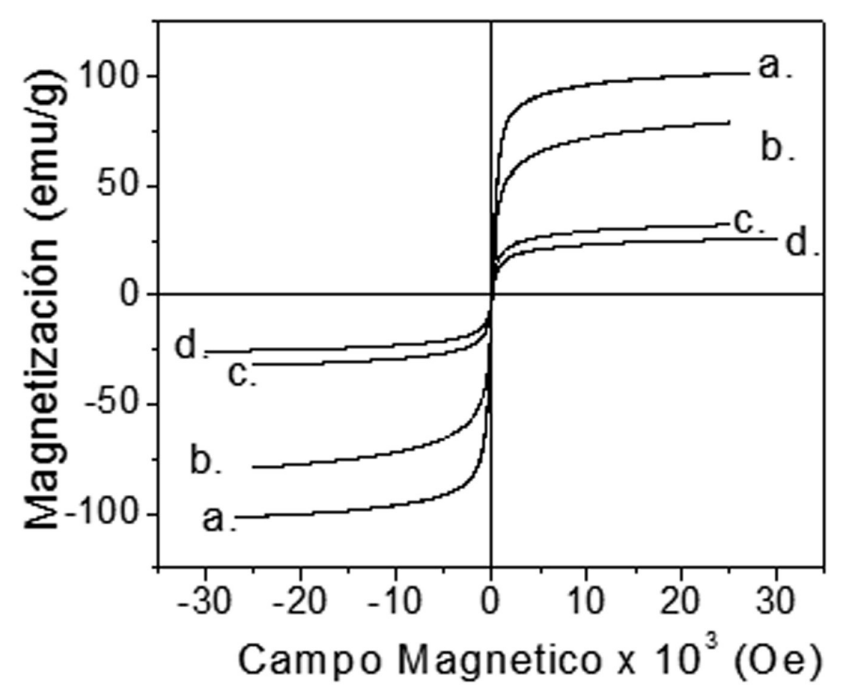

Figura 2. Curvas de magnetización para los soportes magnéticos. (a) Fe3O4, (b) Fe3O4-NH2, (c)Fe3O4@SiO2, (d) Fe3O4@SiO2-NH2. 


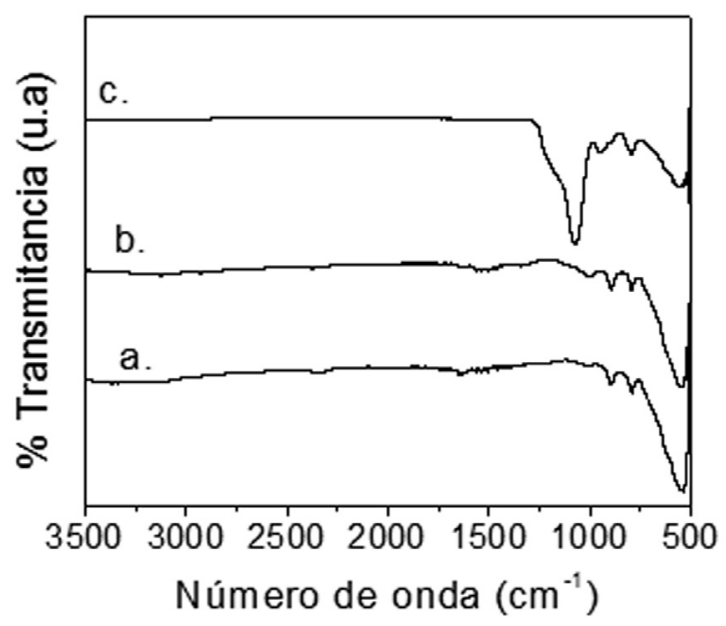

Figura 3. Espectros FTIR, de (a) $\mathrm{Fe}_{3} \mathrm{O}_{4}$, (b) $\mathrm{Fe}_{3} \mathrm{O}_{4}-\mathrm{NH}_{2}$, (c) $\mathrm{Fe}_{3} \mathrm{O}_{4} @ \mathrm{SiO}_{2}-\mathrm{NH}_{2}$.

$\mathrm{cm}^{-1}$, corresponden a las bandas de estiramiento de los grupos $\mathrm{Si}-\mathrm{O}-\mathrm{H}$ y vibraciones de los grupos -OH de la superficie de la magnetita.

\subsection{Evaluación de la proteína acoplada}

Se determinó el nivel de proteína acoplado (PA), por medio de la diferencia entre la proteína del sobrenadante y de la enzima libre $(238 \mu \mathrm{g} /$ $\mathrm{mL}$ ). En la tabla 2, se comparan la cantidad de proteína acoplada en los soportes evaluados, siendo los soportes modificados con APTES, los que mayor cantidad de proteína acoplaron, hecho que se atribuye a que la modificación con APTES asegura una mayor densidad de grupos amino que permite una mayor interacción con los grupos carboxílicos de la proteína. De igual manera, se calculo la proteína acoplada por cantidad de soporte utilizado (PAS), este dato corrobora que la cantidad de soporte no influye en la cantidad de proteína adherida y que esta depende solamente de la cantidad de grupos superficiales del soporte que permiten la adsorción de esta. Frente a los soportes que no se funcionalizaron con APTES, los valores bajos de cantidad proteína acoplada se debe a la lixiviación por las débiles fuerzas electroestáticas que unen a la enzima con el soporte, ocasionando que toda la proteína quedara en el sobrenadante.

\subsection{Evaluación de la actividad enzimática}

Para la actividad enzimática, se evaluó la producción de amoniaco en la hidrolisis de urea catalizada por la enzima por seguimiento colorimétrico a $588 \mathrm{~nm}$. La tabla 3, resume los resultados de actividad enzimática expresada en unidades internacionales (UI) de la enzima inmovilizada y UI/mg de soporte. Estos valores se comparan con el valor de actividad obtenido de la ureasa libre. Puede observarse que no hay relación directa entre proteína acoplada y actividad enzimática, dado que pequeñas cantidades de enzima inmovilizada hidrolizan en un rango similar la urea. Los valores son comparables a la enzima libre $\left(130 \mu \mathrm{M} \mathrm{NH}_{4} \mathrm{OH} / \mathrm{min}\right)$ y la funcionalización con APTES que permite una mayor adsorción de enzima, no necesariamente se refleja en una elevada actividad catalítica. Una explicación del porque no hay una relación directa entre proteína acoplada y actividad catalítica, se explica en términos de la conformación adoptada por la enzima al momento de inmovilizarse. En todos los casos, la inmovilización mejora notablemente la actividad enzimática y los soportes empleados resultan promisorios para la inmovilización de enzimas, lo que permitiría estudiar la cinética y evaluar su aplicabilidad en un proceso industrial.

\section{Conclusiones}

En todos los soportes magnéticos empleados, la inmovilización de la ureasa mejoró notablemente su actividad enzimática. Con una muy pequeña

Tabla 2. Evaluación de proteína acoplada (PA) y acoplada por

\begin{tabular}{ccc}
\multicolumn{3}{c}{$m g$ de soporte $($ PAS $)$} \\
\hline Soporte & $\boldsymbol{\mu M} \mathbf{N H 4 O H}$ min & UI/mg soporte \\
\hline Fe3O4 & 90 & 899 \\
Fe3O4-NH2 & 110 & 1100 \\
Fe3O4@SiO2 & 102 & 1018 \\
Fe3O4@SiO2-NH2 & 137 & 1373 \\
\hline
\end{tabular}


cantidad de ureasa inmovilizada es posible lograr una actividad similar a la de la enzima libre. El comportamiento magnético de los sólidos ofrece una clara posibilidad de fácil separación una vez sean empleados.

\section{Agradecimientos}

Se agradece el soporte financiero de la Dirección de investigaciones de la Universidad Pedagógica y Tecnológica de Colombia, bajo el proyecto SGI N ${ }^{\circ} 1125$.

\section{Referencias bibliográficas}

Ansari, S. A., \& Husain, Q. (2012). Potential applications of enzymes immobilized on/in nano materials: A review. Biotechnology Advances 30 (3), 512-523.

Ashtari, K., Khajeh, K., Fasihi, J., Ashtari, P., Ramazani, A., \& Vali, H. (2012). Silicaencapsulated magnetic nanoparticles: Enzyme immobilization and cytotoxic study. International Journal of Biological Macromolecules 50 (4), 1063-1069.

Bolivar, J. M., Consolati, T., Mayr, T., \& Nidetzky, B. (2013). Shine a light on immobilized enzymes: real-time sensing in solid supported biocatalysts. Trends in Biotechnology 31 (3), 194-203.

Cao, M., Li, Z., Wang, J., Ge, W., Yue, T., Li, R., Colvin, V. L., \& Yu, W. (2012). Food related applications of magnetic iron oxide nanoparticles: Enzyme immobilization, protein purification, and food analysis. Trends in Food Science \& Technology 27 (1), 47-56.

Demir, A. S., Talpur, F. N., Betul Sopaci, S., Kohring, G. W., \& Celik, A. (2011). Selective oxidation and reduction reactions with cofactor regeneration mediated by galactitol, lactate, and formate dehydrogenases immobilized on magnetic nanoparticles. Journal of Biotechnology 152 (4), 176-183.

Díez, P., Villalonga, R., Villalonga, M. L., \& Pingarrón, J. M. (2012). Supramolecular immobilization of redox enzymes on cyclodextrin- coated magnetic nanoparticles for biosensing applications. Journal of Colloid and Interface Science 386 (1), 181-188.

Feng, B., Hong, R., Wang, L. S., Guo, L., Li, H. Z., Ding, J., Zheng, Y., \& Wei, D. G. (2008). Synthesis of $\mathrm{Fe} 3 \mathrm{O} 4 / \mathrm{APTES} / \mathrm{PEG}$ diacid functionalized magnetic nanoparticles for MR imaging. Colloids and Surfaces A: Physicochemical and Engineering Aspects 328 (1-3), 52-59.

Hong, Y., Li, J., Zhang, S., Li, H., Zheng, Y., Ding, J., \& Wei, D.-G. (2009). Preparation and characterization of silica-coated $\mathrm{Fe} 3 \mathrm{O} 4$ nanoparticles used as precursor of ferrofluids. Applied Surface Science 255 (6), 3485-3492.

Jiang, Y., Guo, C., Xia, H., Mahmood, I., Liu, C., \& Liu, H. (2009). Magnetic nanoparticles supported ionic liquids for lipase immobilization: Enzyme activity in catalyzing esterification. Journal of Molecular Catalysis B: Enzymatic 58 (1-4), 103109.

Krishna, L., Singh, N., Patra, S., \& Dubey, K. (2011). Purification, characterization and immobilization of urease from Momordica charantia seeds. Process Biochemistry 46 (7), 1486-1491.

Luo, B., Song, J., Zhang, F., Xia, A., Yang, L., $\mathrm{Hu}, \mathrm{H}$., \& Wang, C. (2010). Multi-functional thermosensitive composite microspheres with high magnetic susceptibility based on magnetite colloidal nanoparticle clusters. Langmuir 26 (3), 1674-1679.

Luo, Z., \& Fu, X. (2010). Immobilization of urease on dialdehyde porous starch. Starch - Stärke 62 (12), 652-657.

Ma, M., Zhang, Y., Yu, W., Shen, H., Zhang, H., $\& \mathrm{Gu}$, N. (2003). Preparation and characterization of magnetite nanoparticles coated by amino silane. Colloids and Surfaces A: Physicochemical and Engineering Aspects 212 (2-3), 219-226.

Netto, M., Toma, E., \& Andrade, H. (2013). Superparamagnetic nanoparticles as versatile carriers and supporting materials for enzymes. 
Journal of Molecular Catalysis B: Enzymatic 8586 (1), 71-92.

Sahoo, B., Sahu, K., \& Pramanik, P. (2011). A novel method for the immobilization of urease on phosphonate grafted iron oxide nanoparticle. Journal of Molecular Catalysis B: Enzymatic 69 (3-4), 95-102.

Soo Y., R. S., Rabolt J., \& Stroeve P. (1996). Synthesis and characterization of nanometer Size $\mathrm{Fe}_{3} \mathrm{O}_{4}$ and $\mathrm{y}-\mathrm{Fe}_{2} \mathrm{O}_{3}$. Chem. Mater 8 (9), 2209-2211.

Tartaj, P., Morales, M. P., González-Carreño, T., Veintemillas-Verdaguer, S., \& Serna, J. (2005). Advances in magnetic nanoparticles for biotechnology applications. Journal of Magnetism and Magnetic Materials 290-291, (4), 28-34.

Tomotani, E. J., \& Vitolo, M. (2006). Catalytic performance of invertase immobilized by adsorption on anionic exchange resin. Process Biochemistry 41 (6), 1325-1331.
Tomotani, E. J., \& Vitolo, M. (2007). Production of high-fructose syrup using immobilized invertase in a membrane reactor. Journal of Food Engineering 80 (2), 662-667.

Waldron, R. D. (1955). Infrared Spectra of Ferrites. Physical Review 99 (6), 1727-1735.

Zhao, G., Wang, J., Li, Y., Chen, X., \& Liu, Y. (2011). Enzymes Immobilized on Superparamagnetic $\mathrm{Fe}_{3} \mathrm{O}_{4} @$ Clays Nanocomposites: Preparation, Characterization, and a New Strategy for the Regeneration of Supports. The Journal of Physical Chemistry C 115 (14), 6350-6359.

Zong, J., Zhu, Y., Yang, X., \& Li, C. (2011). Preparation of monodispersed mesoporous silica spheres with tunable pore size and pore-size effects on adsorption of $\mathrm{Au}$ nanoparticles and urease. Materials Science and Engineering: C 31 (2), 166-172. 